\title{
MODELLING, SIMULATION AND TESTING OF AN OPTOMECHATRONICS DESIGN OF A LARGE RADIO TELESCOPE
}

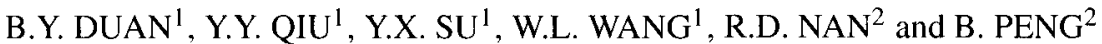 \\ ${ }^{1}$ School of Electro-Mechanical Engineering, Xidian University, Xi' an 710071, China; ${ }^{2}$ Beijing \\ Astronomical Observatory, Chinese Academy of Sciences, Beijing 100012, China
}

\begin{abstract}
An innovative design for a feed support structure for the next generation large radio telescope, based on the idea of integrating mechanical, electronic and optical technologies (OPTOMECHATRONICS), is considered. Theoretical analyses and simulations are carried out. A $5 \mathrm{~m}$ experimental model is built to demonstrate the idea.
\end{abstract}

Keywords: optomechatronics, large radio telescope, nonlinear dynamic analysis, cable

\section{Introduction}

With the development of radio astronomy, astronomers have come to a common point of building a new generation large radio telescope. So far, the largest spherical radio telescope in the world is the Arecibo telescope of $305 \mathrm{~m}$ diameter built in the 1960's and located in Puerto Rico, USA. Due to the technical limitations of the time, the line feed control and tracking system were implemented by mechanical means. The weight of the line feed structure suspended by several long cables is over 800 tons (Mervis and Kinoshiata, 1995). As a result, it is difficult to implement mechanically and also not easy to obtain high tracking precision. This design is probably unacceptably expensive for a square kilometer array element.

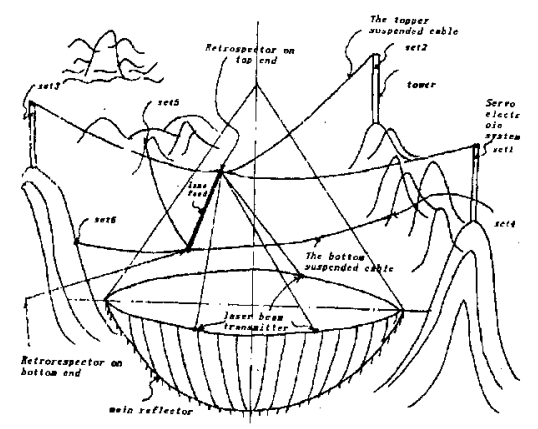

Figure 1. Optomechatronic design of line feed. 


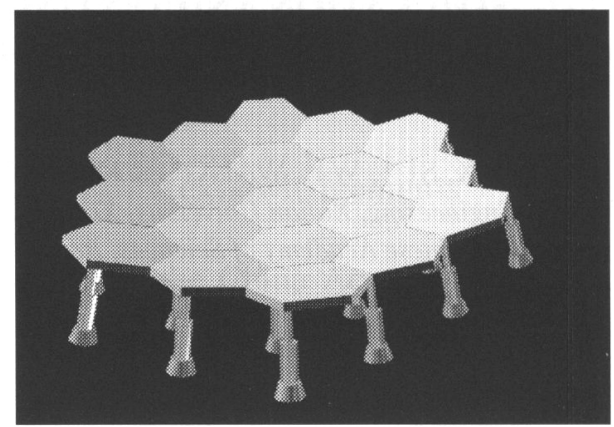

Figure 2. The active reflector of telescope.

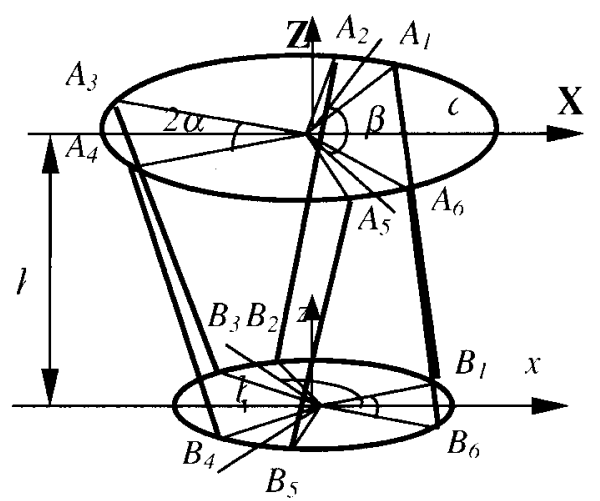

Figure 3. The fine-tuning Stewart stabilizer.

\section{Integrated Design of a Large Radio Telescope with Mechanical, Electronic and Optical Technologies}

Considering the mechanical, electronic, optical and control technologies together, a completely different design (Duan et al., 1995, 1999) from the Arecibo style is proposed (Figure 1). In this project, the 800-ton-weight feed system is discarded and replaced by a line feed of only about 20 tons, and six cables that are suspended from three towers on hills. The six cables are driven by six sets of servo-mechanisms so that movement of the line feed along its trajectory can be realized.

To make sure the six cables move synchronously, a computer controls six sets of servomechanisms. During operation, the theoretical position of the feed will be affected by random loads, for example, the wind. To know its position in time, a laser test system is employed, connected to a computer to create a servo-loop. In this case, not only high tracking accuracy but also a high performance-cost ratio can be achieved. 


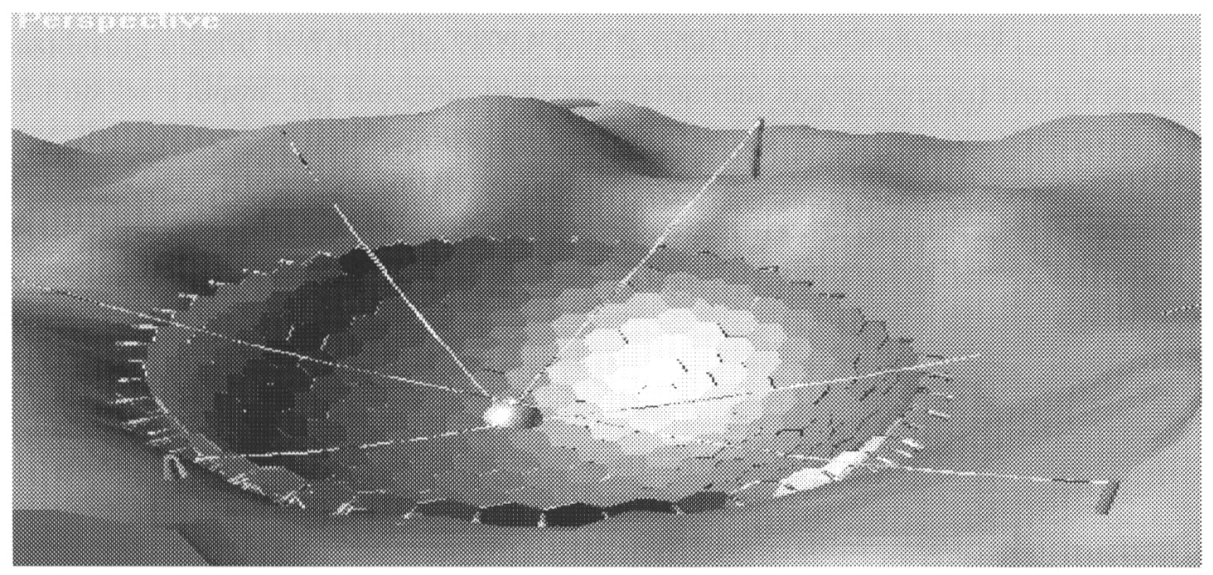

Figure 4. The optomechatronics design with feed cabin.

Even though the key problems, such as cost, weight and engineering implementation, have been solved, there exist two more problems. One is the frequency bandwidth, for it is considerably limited by the line feed. The other is spherical error. To overcome these two problems, Qiu (1998) proposed an active reflector (Figure 2). It consists of many small computer-controlled hexagonal plates. Controlling these plates, a parabolic reflector is simulated. In this case, a point feed instead of the line feed is required. However, it will be very difficult or impossible to obtain millimeter positioning precision of the feed just with long cables. For this reason, a new optomechatronics design (Figure 4) with a cabin and point feed is proposed (Su and Duan, 2000). Here a two level control trajectory is suggested. The cabin is roughly positioned to centimeter accuracy by cables, and then further to millimeter accuracy with a fine-tuning stabilizer (Figure 3). The top plate is fixed to the cabin while the bottom plate can be moved by six line-actuators.

\section{Numerical Simulation}

Numerical simulations have been carried out for the project design (Figure 4). One is a nonlinear response analysis of the cabin-cable system with respect to random wind. The other is a precision analysis of the fine-tuning stabilizer.

\subsection{NONLINEAR RESPONSE ANALYSIS OF CABIN-CABLE SYSTEM TO WIND LOAD}

According to statistical data for the karst formations in the Guizhou area of China, the average wind speed is $1.4 \mathrm{~m} / \mathrm{s}$, the maximum is $17 \mathrm{~m} / \mathrm{s}$ (rare). The data on Puding county between 1991-1997, where many good KARST formations have 
TABLE I

Result of cabin at two typical positions under different wind speed

\begin{tabular}{lcccc}
\hline Wind speed (m/s) & 1.4 & 5.0 & 10 & 17 \\
Maximum displacement (cm) (D1) & 0.07 & 0.7 & 2.0 & 10.5 \\
Maximum displacement (cm) (D2) & & & 9.8 & 52.2 \\
\hline
\end{tabular}
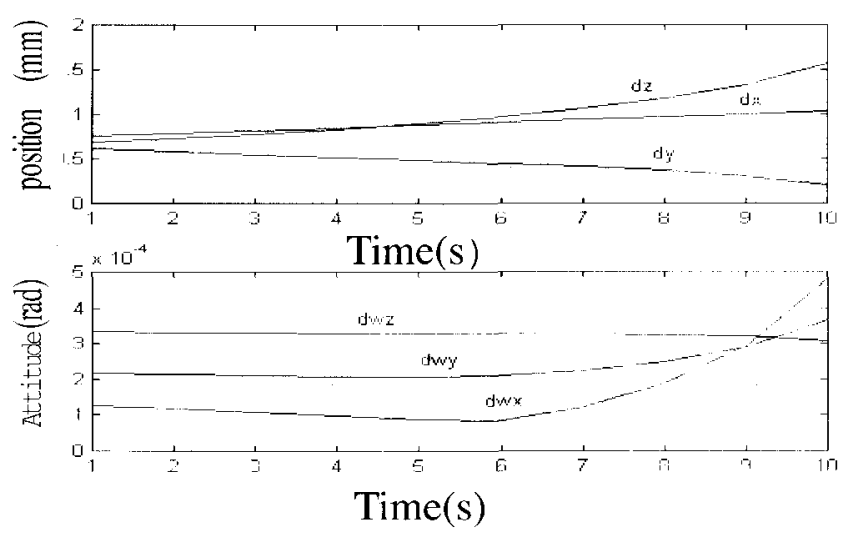

Figure 5. Precision of fine-tuning stabilizer.

been found, has shown that the normal high wind speed is $5 \mathrm{~m} / \mathrm{s}$, with a maximum of $9 \mathrm{~m} / \mathrm{s}$. Therefore, the analysis was carried out for $1.4 \mathrm{~m} / \mathrm{s}, 5 \mathrm{~m} / \mathrm{s}$, and $17 \mathrm{~m} / \mathrm{s}$, respectively. The results without adding additional damping are given in Table I, in which, positions D1 $(-1355.9,0.0,15616.2)(\mathrm{cm})$ and D2 $(-3023.61$, $9526.82,19192.86)(\mathrm{cm})$ are two typical points. D1 and D2 are at the middle and end positions of the trajectory, respectively. Further detailed theoretical deductions can be found in papers (Duan, 1999; Su and Duan, 2000a; Duan, 2000; Su and Duan, 2000b). Obviously, it is possible to achieve a precision of $50 \mathrm{~cm}$ if additional damping is utilized.

\subsection{Precision analysis OF FINE-TUNing Stabilizer}

A study using inverse positional computation, kinematics and singularity analysis is needed to design the Stewart platform and make a precision analysis. These detailed computations can be found in papers ( $S u$ and Duan, 2000a, b). Here just the moving precision is discussed. Suppose $\delta l$ and $\delta p$ are separately the errors of the line actuator and spherical connection between the line actuator and the top and bottom plates. $\delta x$ and $\delta \Omega$ are the position and attitude errors of the movable plate. $\delta x$ and $\delta \Omega$ can be found from $\delta l$ and $\delta p$.

The vibration computation is based on the condition that the movable plate load is 4 tons, the required velocity is $0.5,0.5,0.5,0.0035,0.0035$ and 0.0035 
$(\mathrm{cm} / \mathrm{s}$ and $\mathrm{rad} / \mathrm{s}$ for the former and latter three components, respectively), without considering elastic deformation of the line actuator. By selecting $|\delta l|=1.008 \mathrm{~mm}$ and $|\delta p|=1.0 \mathrm{~mm}$, the movable plate precision can be determined from Figure 5 . Since the accessible region must be covered within a certain time interval, the precision-curve time axis shows in detail how the feed tracks the target. For the assumed velocity, 10 seconds is sufficient to cover the required space. Therefore, the precision history over 10 seconds is described by Figure 5. Clearly, millimeter precision can be obtained.

\section{A 5-m Experimental Model}

In order to demonstrate the design project and pave a solid path to constructing a $50 \mathrm{~m}$ demonstration model, and ultimately a $500 \mathrm{~m}$ telescope, a $5 \mathrm{~m}$ model has been built with success at the research institute of mechatronics at Xidian University.

\section{Conclusion}

The design project, with the integration of mechanical, electronic, optical and control technologies, can be considered a revolutionary design compared to the Arecibo telescope, in which some key difficulties faced when building a new generation large radio telescope can, to a certain extent, be overcome.

Detailed analysis of the cable and cabin system and the fine-tuning stabilizer has shown that the optomechatronics design is capable of satisfying the precision required. The precision can be realized by both rough control $(\mathrm{cm}$ level) of the cable and cabin and a fine-tuning stabilizer control ( $\mathrm{mm}$ level).

A $5 \mathrm{~m}$ model was made to precede the $50 \mathrm{~m}$ model, from which the key technologies have been demonstrated, such as control, detection and so on. This paves the way to constructing the $50 \mathrm{~m}$ model as well as to the $500 \mathrm{~m}$ FAST project engineering. In addition to simulation, experiments are badly needed to test key technologies, which may be the main obstacle on the way to finally constructing FAST.

\section{References}

Duan, B.Y., et al: 1995, Proceedings of The Third International Conference On Large Radio Telescope, Guizhou of China, October 2-6.

Duan, B.Y.: 1999, A new design project of the line feed structure for large spherical radio telescope and its nonlinear dynamic analysis, Int. J. Mechatronics 9(1), 53-64.

Duan, B.Y.: 2000, Study on next generation large radio telescope, Report at Jordrell Bank Astronomy Observatory, Manchester University, United Kingdom, January 23-26.

Mervis, J. and Kinoshiata, J.: 1995, J. Science Amer. 270 November. 
Qiu, Y.H.: 1998, Novel design for the giant spherical radio telescope - Arecibo-type with an active main reflector, Beijing Astron. Observ. March.

Su, Y.X. and Duan, B.Y.: 2000a, The mechanical design and kinematics accuracy analysis of a finetuning stable platform for the large spherical radio telescope, Int. J. Mechatronics, 10(7), 819834.

Su, Y.X. and Duan, B.Y.: 2000b, Application of Stewart platform in the next generation large radio telescope, Int. J. Robotic Syst. 17(7), 375-383. 